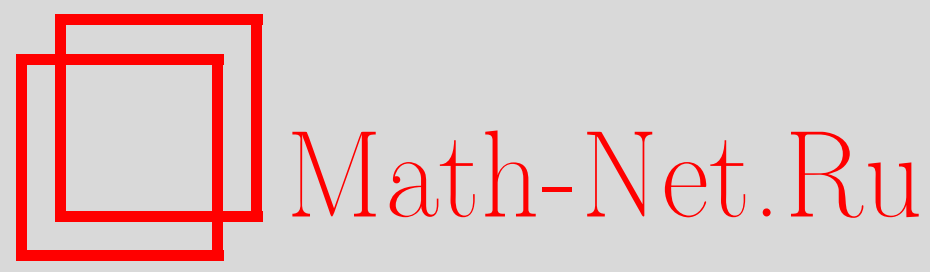

М. Е. Лернер, О. А. Репин, Существенно нелокальная краевая задача для уравнения с частными производными, $\mathrm{Ma}$ тем. заметки, 2000, том 67, выпуск 3, 478-480

DOI: https://doi.org/10.4213/mzm861

Использование Общероссийского математического портала Math-Net.Ru подразумевает, что вы прочитали и согласны с пользовательским соглашением http://www . mathnet.ru/rus/agreement

Параметры загрузки:

IP : 54.198 .187 .58

26 апреля 2023 г., $11: 27: 14$

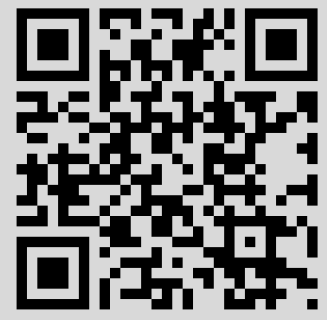




\section{СУЩЕСТВЕННО НЕЛОКАЛЬНАЯ КРАЕВАЯ ЗАДАЧА ДЛЯ УРАВНЕНИЯ С ЧАСТНЫМИ ПРОИЗВОДНЫМИ}

\section{М. Е. Лернер, О.А. Репин}

В отличие от известных нелокальных краевых задач для уравнений с частньми производными [1]-[5] в данной работе ставится задача, содержащая только нелокальные краевые условия (что отражено в названии статьи).

Рассмотрим уравнение Геллерстедта

$$
G_{m} u \equiv \operatorname{sgn} y|y|^{m} u_{x x}+u_{y y}=0, \quad m>0,
$$

в области $D$, ограниченной полубесконечными прямыми $O O_{\infty}(O(0 ; 0), x=0, y \geqslant 0)$ и $A A_{\infty}$ $(A(1 ; 0), x=1, y \geqslant 0)$ и характеристиками уравнения (1) $O C$ и $A C, C\left(1 / 2,-((m+2) / 4)^{2 /(m+2)}\right)$.

Пусть $D_{1}=D \cap\{(x, y): y>0\}, D_{2}=D \cap\{(x, y): y<0\}, O_{h}(0, h), A_{h}(1, h), h>0 ; D_{1_{h}}-$ открытый прямоугольник с вершинами $O, O_{h}, A_{h}, A ; D_{h}=D_{1_{h}} \cup O A \cup D_{2}$.

ЗАДАчА 1 . Найти функцию $u(x, y)$ со свойствами:

1) $G_{m} u \equiv 0$ в $D_{1} \cup D_{2}$;

2) $u \in C^{0}(\bar{D}) \cap C^{1}(\bar{D} \backslash \overline{O A}) \cap C^{2}(D \backslash O A)$;

3) $\lim _{y \rightarrow+\infty} u(x, y)=0$ равномерно по $x \in[0,1]$;

4) существуют конечные и равные между собой пределы

$$
\lim _{y \rightarrow 0-0} u_{y}(x, y)=\lim _{y \rightarrow 0+0} u_{y}(x, y), \quad 0<x<1
$$

5) $u(0,0)=0$;

6) $u(0, y)-u(1, y)=\varphi_{1}(y), y \geqslant 0$;

7) $u_{x}(0, y)-u_{x}(1, y)=\varphi_{2}(y), y>0$;

8) $a(x)\left(I_{0+}^{\beta-1,1-\beta, 1-\beta} u\left[\Theta_{0}(t)\right]\right)(x)+b(x)\left(I_{1-}^{\beta-1,1-\beta, 1-\beta} u\left[\Theta_{1}(t)\right]\right)(x)=c(x), x \in[0,1]$, где $\varphi_{1}(y), \varphi_{2}(y), a(x), b(x), c(x)$ - заданные функции такие, что

$$
\varphi_{1}(y) \in C^{0}[0,+\infty) \cap C^{\prime}(0,+\infty), \quad \lim _{y \rightarrow+\infty} \varphi_{1}(y)=0, \quad \varphi_{2}(y) \in C^{0}(0,+\infty),
$$

$a(x), b(x), c(x) \in C^{0}[0,1], \quad(1-x)^{\beta} a(x)+x^{\beta} b(x) \neq 0 \quad$ для всех $x \in[0,1], \quad \beta=\frac{m}{2(m+2)}$,

$$
\Theta_{0}(x)=\frac{x}{2}-i\left(\frac{m+2}{4} x\right)^{2 /(m+2)}, \quad \Theta_{1}(x)=\frac{1+x}{2}-i\left(\frac{m+2}{4}(1-x)\right)^{2 /(m+2)},
$$

$\Theta_{0}$ и $\Theta_{1}-$ афффиксы точек пересечения характеристик уравнения (1), выходящих из точки $(x, 0)$, с характеристиками $O C$ и $A C$ соответственно, $\left(I_{0+}^{\alpha, \beta, \eta} f\right)(x)$ и $\left(I_{1-}^{\alpha, \beta, \eta} f\right)(x)-$ операторы обобщенного дробного интегродифференцирования, определяемые формулами (см. [6], [7])

$$
\begin{aligned}
& \left(I_{0+}^{\alpha, \beta, \eta} f\right)(x)=\left\{\begin{array}{l}
\frac{x^{-\alpha-\beta}}{\Gamma(\alpha)} \int_{0}^{x}(x-t)^{\alpha-1} F\left(\alpha+\beta,-\eta ; \alpha ; 1-\frac{t}{x}\right) f(t) d t, \quad \alpha>0, \\
\frac{d^{n}}{d x^{n}}\left(I_{0+}^{\alpha+n, \beta-n, \eta-n} f\right)(x), \quad \alpha \leqslant 0, n=[1-\alpha],
\end{array}\right. \\
& \left(I_{1-}^{\alpha, \beta, \eta} f\right)(x)=\left\{\begin{array}{l}
\frac{(1-x)^{-\alpha-\beta}}{\Gamma(\alpha)} \int_{x}^{1}(t-x)^{\alpha-1} F\left(\alpha+\beta,-\eta ; \alpha ; \frac{t-x}{1-x}\right) f(t) d t, \quad \alpha>0, \\
(-1)^{n} \frac{d^{n}}{d x^{n}}\left(I_{1-}^{\alpha+n, \beta-n, \eta-n} f\right)(x), \quad \alpha \leqslant 0, n=[1-\alpha] .
\end{array}\right.
\end{aligned}
$$


Операторы (2), (3) при $\beta=-\alpha$ и $\beta=0$ сводятся к дробным интегралам и производным Римана-Лиувилля и Кобера [5], [8] соответственно.

Заметим, что нелокальое условие 8) может быть записано с помощью операторов Римана-Лиувилля (см. [5], [9])

$$
a(x) D_{0+}^{1-\beta} u\left[\Theta_{0}(t)\right]+b(x) D_{1-}^{1-\beta} u\left[\Theta_{1}(t)\right]=c(x) .
$$

Однако, прежняя его форма оказалась более удобной для цели данного исследования.

1. Рассмотрим уравнение (1) в области $D_{2}$. Пусть $u(x, y)$ - решение задачи $1, u(x, 0)=\tau(x)$, $u_{y}(x, 0)=\nu(x)$. Установим связь между $\tau(x)$ и $\nu(x)$. Если $\tau(x) \in C^{0}[0,1] \cap C^{2}(0,1)$ и $\nu(x) \in$ $C^{1}(0,1)$, то в области $D_{2}$ функцию $u(x, y)$ можно представить по формуле решения задачи Коши (в переменных $\xi, \eta)[9]$ :

$$
u(\xi, \eta)=\gamma_{1} \int_{\xi}^{\eta}(\eta-\xi)^{1-2 \beta}(\eta-t)^{\beta-1}(t-\xi)^{\beta-1} \tau(t) d t-\gamma_{2} \int_{\xi}^{\eta}(\eta-t)^{-\beta}(t-\xi)^{-\beta} \nu(t) d t
$$

где

$$
\xi, \eta=x \mp \frac{2}{2+m}(-y)^{(2+m) / 2}, \quad \gamma_{1}=\frac{\Gamma(2 \beta)}{\Gamma^{2}(\beta)}, \quad \gamma_{2}=\frac{1}{2}\left(\frac{4}{2+m}\right)^{2 \beta} \frac{\Gamma(1-2 \beta)}{\Gamma^{2}(1-\beta)} .
$$

Из (4), используя (2) и (3), имеем

$$
\begin{aligned}
& u\left[\Theta_{0}(x)\right]=\gamma_{1} \Gamma(\beta)\left(I_{0+}^{\beta, \beta-1,0} t^{\beta-1} \tau\right)(x)-\gamma_{2} \Gamma(1-\beta)\left(I_{0+}^{1-\beta, \beta-1,0} t^{-\beta} \nu\right)(x), \\
& u\left[\Theta_{1}(x)\right]=\gamma_{1} \Gamma(\beta)\left(I_{1-}^{\beta, \beta-1,0}(1-t)^{\beta-1} \tau\right)(x)-\gamma_{2} \Gamma(1-\beta)\left(I_{-1}^{1-\beta, \beta-1,0}(1-t)^{-\beta} \nu\right)(x) .
\end{aligned}
$$

Подставляя (5) и (6) в краевое условие 8), на основании соотношений [7]

$$
\begin{array}{ll}
\left(I_{0+}^{\alpha, \beta, \eta}\left(I_{0+}^{\gamma, \delta, \alpha+\eta} f\right)(t)\right)(x)=\left(I_{0+}^{\alpha+\gamma, \beta+\delta, \eta} f\right)(x), & \gamma>0, \\
\left(I_{1-}^{\alpha, \beta, \eta}\left(I_{1-}^{\gamma, \delta, \alpha+\eta} f\right)(t)\right)(x)=\left(I_{1-}^{\alpha+\gamma, \beta+\delta, \eta} f\right)(x), & \gamma>0,
\end{array}
$$

получим связь между $\tau(x)$ и $\nu(x)$ :

$$
\begin{aligned}
& a(x) \gamma_{1} \Gamma(\beta)\left(I_{0+}^{2 \beta-1,0,1-\beta} t^{\beta-1} \tau\right)(x)-a(x) \gamma_{2} \Gamma(1-\beta) x^{-\beta} \nu(x) \\
& \quad+b(x) \gamma_{1} \Gamma(\beta)\left(I_{1-}^{2 \beta-1,0,1-\beta}(1-t)^{\beta-1} \tau\right)(x)-b(x) \gamma_{2} \Gamma(1-\beta)(1-x)^{-\beta} \nu(x)=c(x) .
\end{aligned}
$$

Из (7) при $c(x) \equiv 0$ следует, что

$$
\left[(1-x)^{\beta} a(x)+x^{\beta} b(x)\right] \nu(x)=\frac{\gamma_{1} \Gamma(\beta)}{\gamma_{2} \Gamma(1-\beta)}\left[(1-x)^{\beta} a(x)\left(D_{0+}^{1-2 \beta} \tau\right)(x)+x^{\beta} b(x)\left(D_{1-}^{1-2 \beta} \tau\right)(x)\right],
$$

где $\left(D_{0+}^{\alpha} f\right)(x)$ и $\left(D_{1-}^{\alpha} f\right)(x)$ - операторы дробного интегродифференцирования в смысле РиманаЛиувилля [8]. Из (8) непосредственно вытекает следующее утверждение.

Лемма 1. $\operatorname{Ecли~} c(x) \equiv \tau(x) \equiv 0$ на $[0,1]$, то $\nu(x) \equiv 0$ на $[0,1]$.

ЛЕмма 2. Пусть

$$
\max _{[0,1]} \tau(x)=\tau\left(x_{0}\right)>0 \quad\left(\min _{[0,1]} \tau(x)=\tau\left(x_{0}\right)<0\right),
$$

$x_{0} \in(0,1), a(x) b(x) \geqslant 0, c(x) \equiv 0$ на $[0,1]$. Тогда $\nu\left(x_{0}\right)>0\left(\nu\left(x_{0}\right)<0\right)$.

Доказательство леммы следует из (8) и принципа максимума для производных дробного порядка [9], [10].

2. Рассмотрим теперь уравнение (1) в области $D$.

Tеорема 1. Решение задачи 1 единственно в классе функиий $u(x, y) \in C^{0}(\bar{D}) \cap C^{1}(\bar{D} \backslash \overline{O A})$ $\cap C^{2}(D \backslash O A)$ и представимого в области $D_{2}$ по формуле (4) решения задачи Коши, если $a(x), b(x), c(x) \in C^{0}[0,1]$. 
ДоКАЗАТЕЛЬСтво. Пусть $u(x, y)$ - решение однородной задачи 1 и, в частности,

$$
\begin{aligned}
u(0, y) & =u(1, y), \quad y \geqslant 0, \\
u_{x}(0, y) & =u_{x}(1, y), \quad y>0 .
\end{aligned}
$$

Покажем, что $u(x, y) \equiv 0$ в области $D_{1}$. Предположим противное. Тогда найдется область $D_{1_{h}}$, в которой $u(x, y) \not \equiv 0$ и, следовательно,

$$
\max _{\bar{D}_{h}}|u|>0 \text {. }
$$

Без ограничения общности можно считать, что

Убедимся, что

$$
\overline{\max }_{1_{h}}|u|=\overline{\max }_{1_{h}} u>0 .
$$

$$
\overline{\max }_{1_{h}} u
$$

достигается на $\overline{O_{h} A_{h}}$. Допустим, что это не так. Следовательно, он должен достигаться на $\overline{O_{h} O} \cup$ $O A \cup \overline{A A_{h}}$. Пусть (11) достигается на $O O_{h}$ в некторой точке $\left(0, y_{0}\right)$. Тогда ввиду $(9)$ он должен достигаться на $A A_{h}$ в точке $\left(1, y_{0}\right)$. В силу хорошо известного свойства эллиптических уравнений $u_{x}\left(0, y_{0}\right)<0$ и $u_{x}\left(1, y_{0}\right)>0$, что противоречит (10). Так же (11) не может достигаться в точках $O$ и $A$ ввиду условия 7$)$ задачи 1 .

Таким образом, (11) с учетом сделанного предположения не может достигаться на $\overline{O O_{h}} \cup O_{h} A_{h}$ $\cup \overline{A_{h} A}$ и, следовательно, должен достигаться в некоторой внутренней точке $\left(x_{0}, 0\right)$ отрезка $O A$. Но тогда по лемме типа леммы К. И. Бабенко [11], [12] должно быть $u_{y}\left(x_{0}, 0+0\right)<0$, что противоречит лемме 2. Следовательно, (11) должен достигаться на $\overline{O_{h} A_{h}}$. Точно так же можно убедиться, что

$$
\overline{\max }_{1_{h}^{*}} u=\frac{\max }{O_{h^{*}} A_{h^{*}}}|u|
$$

при любом $h^{*}>h$.

Так как $\bar{D}_{1_{h}} \subset \bar{D}_{1_{h^{*}}}$, имеем

$$
\max _{D_{1_{h}}}|u| \geqslant \max _{D_{1_{h}}}|u|>0 .
$$

Отсюда

$$
\lim _{y \rightarrow+\infty} u(x, y) \neq 0,
$$

что противоречит условию 3 ) задачи 1 . Следовательно, $u(x, y) \equiv 0$ в $\bar{D}_{1}$; в частности, $u(x, 0)=$ $\tau(x) \equiv 0$ на $\overline{O A}$. Но тогда $u(x, y) \equiv 0$ в $\bar{D}_{2}$ на основании леммы 1 . Таким образом, $u(x, y) \equiv 0$ в области $D$. Тем самым, теорема 1 доказана.

\section{СПИСОК ЦИТИРОВАННОЙ ЛИТЕРАТУРЫ}

1. Франкль Ф. И. // ПММ. 1956. Т. 20. № 2. С. 196-202. 2. ЖКегалов В. И. // Ученые записки Казанского ун-та. 1962. Т. 122. Кн. 3. С. 3-16. 3. Ионкин Н. И., Моисеев Е. И. // Дифоференц. уравнения. 1979. Т. 15. № 7. С. 1284-1295. 4. Лернер М. Е. // Дифференц. уравнения и матем. физика. Республ. сб. Т. 239. Вьп. 1. Куйбышев, 1979. С. 50-61. 5. Нахушев А. М. Уравнения математической биологии. М.: Высшая школа, 1995. 6. Saigo M. // Math. Japon. 1979. V. 24. № 4. Р. 377-385. 7. Репин О. А. Краевые задачи со смещением для уравнений гиперболического и смешанного типов: Изд-во Саратовского ун-та (Самарский филиал), 1992. 8. Самко С. Г., Килбас А.А., Маричев О.И. Интегралы и производные дробного порядка и некоторые их приложения. Минск: Наука и техника, 1987. 9. Смирнов М. М. Уравнения смешанного типа. М.: Высшая школа, 1985. 10. Нахушев А. М. // Дифференц. уравнения. 1974. Т. 10. № 1. С. 100-111. 11. Бабенко К. И. К проблеме уравнений смешанного типа. Дисс. . . . д. ф.-м. н. М., 1951. 12. Лернер М. Е., Репин О. А. // Математическое моделирование и краевые задачи. Тр. седьмой межвуз. конф. Ч. 3. Самара, 1977. С. 50-54. 